\title{
A efetividade da bandagem elástica funcional nas disfunções pélvicas femininas: uma revisão sistemática de ensaios clínicos randomizados e controlados
}

\author{
The effectiveness of funcional elastic bandagem in fenale pelvic dysfunction: a systematic review of \\ randomized and controlled clinical trials
}

La eficacia del vendaje elastico funcional em las disfunciones pelvicas femeninas: uma revisión sistemática de ensayos clínicos aleatorizados y controlados

Recebido: 12/08/2021 | Revisado: 21/08/2021 | Aceito: 26/08/2021 | Publicado: 29/08/2021

\author{
Amanda Silva Pereira \\ ORCID: https://orcid.org/0000-0002-8905-9468 \\ Universidade Federal do Delta do Parnaíba, Brasil \\ E-mail: amandapereirasilva17@ outlook.com \\ Kryzia de Moraes Azevedo \\ ORCID: https://orcid.org/0000-0002-9183-0645 \\ Universidade Federal do Delta do Parnaíba, Brasil \\ E-mail: kryziaazevedo@gmail.com \\ Williams Silva Lima \\ ORCID: https://orcid.org/0000-0002-4756-8213 \\ Universidade Federal do Delta do Parnaíba, Brasil \\ E-mail: williamssilva@ufpi.edu.br \\ Mona Indianara da Costa Aragão \\ ORCID: https://orcid.org/0000-0003-2557-9912 \\ Universidade Federal do Delta do Parnaíba, Brasil \\ E-mail: monna.aragao@gmail.com \\ Sabrina Amorim Correia \\ ORCID: https://orcid.org/0000-0002-7527-3877 \\ Universidade Federal do Delta do Parnaíba, Brasil \\ E-mail: sabrinna.amorim7@gmail.com \\ Sylvia Christina Leite Gomes \\ ORCID: https://orcid.org/0000-0003-1507-3838 \\ Universidade Federal do Delta do Parnaíba, Brasil \\ E-mail: sylvia.clg@gmail.com \\ Sávia Francisca Lopes Dias \\ ORCID: https://orcid.org/0000-0002-4490-8331 \\ Universidade Federal do Delta do Parnaíba, Brasil \\ E-mail: saviadias@ufpi.edu.br
}

\section{Resumo}

Essa revisão tem como objetivo reunir evidências para identificar a efetividade da bandagem elástica funcional nas DAPs. O levantamento de dados foi realizado utilizando as bases de dados: Medline, Pubmed, Lilacs, SCIELO, PEDRO. O acrômio PICO foi usado para definir as questões da pesquisa, com estudos de no máximo 5 anos. Foram selecionados 9 artigos, separados por área. Obstetrícia (6), Ginecologia (2) e Uroginecologia (1) que estavam dentro dos critérios de inclusão para a realização da revisão sistemática. Concluiu-se que a Bandagem Elástica Funcional atua de maneira eficaz no tratamento das disfunções pélvicas femininas, promovendo bons resultados clínicos em relação dor, ansiedade, qualidade de vida e outros sintomas associados às DAPs.

Palavras-chave: Bandagem elástica funcional; Disfunções do assoalho pélvico; Obstetrícia; Ginecologia; Uroginecologia; Fisioterapia.

\begin{abstract}
This review aims to gather evidence to identify the effectiveness of funcional elastic bandagem in PADs. Data collection was carried out using the following databases: Medline, Pubmed, Lilacs, SciELO and PEDRO. The PICO acromion was used to define the research questions, with studies of a maximum of 5 years. Nine articles were selected, separated by area. Obstetrics (6), Gynecology (2) and Urogynecology (1) which were within the inclusion criteria for the systematic review. It was concluded that the Functional Elastic Bandage works effectively in the treatment of female pelvic disorders, promoting good clinical results in relation to anxiety, quality of life and other symptoms associated with PADs.
\end{abstract}

Keywords: Functional elastic bandagem; Pelvic floor disorders; Obstetrics; Gynecology; Urogynecology; Physiotherapy.

\section{Resumen}

Esta revisión tiene como objetivo reunir pruebas para identificar la efectividad del vendaje elástico funcional en em las EAP. La recolección de datos se realizo utilizando las siguientes bases de datos: Medline, Pubmed, Lilacs, SCIELO, PEDRO. Se 
utilizo el acromion PICO para definir las preguntas de investigación, com estúdios de um máximo de 5 ãnos. Se seleccionaron nueve artículos, separados por áreas. Obstetricia (6), Ginecología (2) y Uroginecologia (1) que se encontraban dentro de los critérios de inclusión para la revisión sistemática. Se concluyó que el Vendaje Elástico Funcional actúa eficazmente em el tratamento de los transtornos pélvicos femininos, promovendo Buenos resultados clínicos em relación al dolor, ansiedade, calidad de vida y otros sintomas associados a las EAP.

Palavras clave: Vendaje elástico funcional; Trastomos del suelo pélvico; Obstericia; Ginecologia; Uroginecologia; Fisioterapia.

\section{Introdução}

A pelve humana é estruturada pelos ossos ílio, ísquio, púbis, sacro e cóccix. Está localizada na parte inferior da coluna vertebral e apresenta duas bases, uma "maior", mais superior, que é ocupada pelas vísceras abdominais, e continua inferiormente até a base mais estreita chamada "menor". A abertura pélvica inferior é fechada pelo assoalho pélvico (AP), este por sua vez, é constituído por músculos e fáscias que agem em conjunto (Palma, Berghmans e Seleme, 2014).

A sustentação muscular da cavidade pélvica ocorre pela contração intencional dos músculos do assoalho pélvico (MAP) em que o levantador do ânus, com seus três feixes puborretal, pubococcígeo e ileococcígeo, realiza levantamento e compressão ao redor da uretra, vagina e ânus, oferecendo suporte estrutural horizontal aos órgãos pélvicos (Resende et al., 2010).

As disfunções do assoalho pélvico (DAPs) acometem um número crescente de mulheres a cada ano (Korelo et al., 2011). Devido à função e estrutura do AP está diretamente relacionada às condições uroginecológicas e obstétricas, as principais DAPs incluem: incontinência urinária (IU), constipação funcional relacionada à dissinergia abdomino-pélvica, prolapsos de órgãos pélvicos (POPs), incontinência anal, dismenorreia, dor pélvica e disfunções sexuais (Arbuckle et al., 2018). Desse modo, acometem aproximadamente um terço da população feminina adulta ao longo da vida, possuindo um impacto negativo na qualidade de vida de muitas mulheres (Silva et al., 2017).

A fisioterapia previne e trata limitações e incapacidades físicas, restaura função, mobilidade e promove alívio da dor. Nas desordens pélvicas, atua na prevenção e tratamento das disfunções dos diversos sistemas, urológico, fecal, ginecológico e sexual (Lemos et al., 2015). Também pode atuar como coadjuvante no tratamento de outras disfunções do assoalho pélvico, potencializando os resultados e melhorando a qualidade de vida dos pacientes (Stein et al., 2018).

Dentre os recursos alternativos para o tratamento das DAPS, encontra-se a bandagem elástica funcional, que é um recurso terapêutico de baixo custo, não invasivo, e com efeito contínuo enquanto a fita estiver sobre a pele da paciente. Esta técnica é fundamentada no princípio da melhora da capacidade de recuperação natural para que haja a homeostase, corrigindo o equilíbrio do corpo humano, ajustando os fluxos eletromagnéticos sobre a pele e estimulando indiretamente músculos ou órgãos que estão logo abaixo da pele (Lenzi et al., 2017).

Desse modo, os princípios básicos da atuação da bandagem elástica na musculatura são: correção da função motora de músculos fracos, aumento da circulação sanguínea e linfática e aumento da propriocepção através da estimulação dos mecanorreceptores cutâneos. A aplicação da bandagem elástica pode aumentar o controle motor, assim como ativar a musculatura desejada (Sordi et al., 2018). Portanto, essa revisão tem como objetivo reunir evidências para identificar a efetividade da bandagem elástica funcional nas disfunções pélvicas femininas.

\section{Metodologia}

O presente estudo é uma revisão sistemática, um tipo de pesquisa que é uma investigação científica, com métodos sistemáticos pré-definidos que buscam identificar sistematicamente todos os documentos relevantes acerca de um determinado tema. Onde são avaliados a qualidade desses artigos, extraídos os dados e sintetizado os resultados (Siddaway et al.,2019). 
O presente estudo é uma revisão sistemática, um tipo de pesquisa que é uma investigação científica, com métodos sistemáticos pré-definidos que buscam identificar sistematicamente todos os documentos relevantes acerca de um determinado tema. Onde são avaliados a qualidade desses artigos, extraídos os dados e sintetizado os resultados (Siddaway et al.,2019).

O levantamento de dados foi realizado utilizando as bases de dados: Medline, Pubmed, Lilacs, SciELO, PEDRO. Foi utilizado como descritores: Fisioterapia; saúde da mulher; obstetrícia; ginecologia; bandagem elástica; assoalho pélvico; urologia. Para a busca de documentos em outro idioma, foram pesquisados esses termos na sua equivalência ao inglês e espanhol, sendo considerados válidos os documentos que apresentassem o cruzamento dessas palavras no título e/ou resumo. Para a definição da questão de pesquisa de forma estruturada foi utilizado o formato do acrônimo PICO, onde cada letra representa um componente da questão, $\mathrm{P}$ (população); I (intervenção); C(controle) e O (desfecho).

Diante do maior número de artigos publicados em periódicos nacionais e internacionais a partir de 2010, percebe-se que houve um aumento da produção científica ocorrido nos últimos anos, na qual, contribuiu para a difusão de uma metodologia potencialmente útil para todos (Strehl et al., 2016). Dessa forma, o período definido inicialmente nas buscas por estudos, foi de 10 anos, posteriormente foi alterado para 5 anos em busca de artigos acerca do tema com metodologia mais bem definida. Sendo assim, durante a pesquisa foram avaliados estudos recentes, com prazo máximo de 5 anos de publicação sem restrição de idioma. O processo de busca de publicações apropriadas foi documentado pelos Itens de Relatórios Preferenciais para Revisões Sistemáticas e Meta-Análise (PRISMA).

A seleção dos artigos para o refinamento da pesquisa e a avaliação dos títulos e dos resumos (abstracts) identificado na busca inicial, foi realizada por dois pesquisadores de forma independente e cegada, obedecendo rigorosamente aos critérios de inclusão como segue: Pesquisa com seres humanos, exclusivamente mulheres que apresentem disfunções pélvicas, relacionadas às áreas: Uroginecológicas e/ou obstétricas, como exemplo: Incontinência urinária, Dismenorreia e Dor Pélvica e foi utilizada como intervenção a bandagem elástica funcional. Seguindo a linha de pesquisa, os critérios de exclusão para o presente estudo foram: artigos de revisão, descritivos de estudos de caso, séries de casos, estudos observacionais e qualitativos.

$\mathrm{Na}$ tabulação dos dados, os resultados foram expressos em um quadro comparativo com a descrição dos autores, data e detalhes da intervenção. Para os artigos foi construído um quadro com a descrição dos principais resultados alcançados, o objetivo, o valor de significância das técnicas empregadas e principais resultados.

\section{Resultados e Discussão}

De acordo com as diretrizes metodológicas para elaboração de revisão sistemática, a definição da pesquisa clínica deve ser estruturada utilizando o acrônimo PICO. A figura 1 descreve a questão desta pesquisa seguindo os componentes do acrônimo PICO (Figura 1).

Figura 1: Definição da questão de pesquisa de acordo com o Acrônimo PICO.

1. População - Mulheres com disfunções pélvicas;

2. Intervenção - Bandagem elástica funcional nas disfunções pélvicas feminina. Tais como: dismenorreia primaria, dor lombar em gestantes e incontinência urinária.

3. Controle - Considerou-se controle positivo: intervenções farmacológicas, exercícios, massagem; controle negativo: nenhuma intervenção; placebo: aplicação de bandagem sem tensão;

4. Desfecho - A bandagem elástica funcional é uma intervenção de baixo custo e sem efeitos colaterais, que apresenta efeitos positivos nas disfunções pélvicas femininas.

Fonte: Autores 
Na busca inicial, sem restrição de idiomas nas bases de dados, foram encontrados 206 artigos. Após nova seleção, foram excluídas as duplicatas e obtidos 184 estudos, dos quais sob outra triagem, foram excluídos mais 70 artigos que se enquadraram como resumos, estudos de caso, séries de casos, artigos de revisão bibliográfica, estudos não controlados, estudos com animais, estudos observacionais e qualitativos. Após isso, um quantitativo de 114 artigos foi avaliado para elegibilidade, no qual 105 estudos foram analisados e excluídos por motivos de não possuir grupo controle, não ser randomizado ou não estar associados à fisioterapia. Por fim, 9 artigos foram avaliados e considerados relevantes para esse trabalho, e nenhum registro foi encontrado por meio de outras fontes. Todas essas informações estão descritas no diagrama de fluxo PRISMA (Figura 2).

Figura 2: Diagrama de Fluxo Prisma.

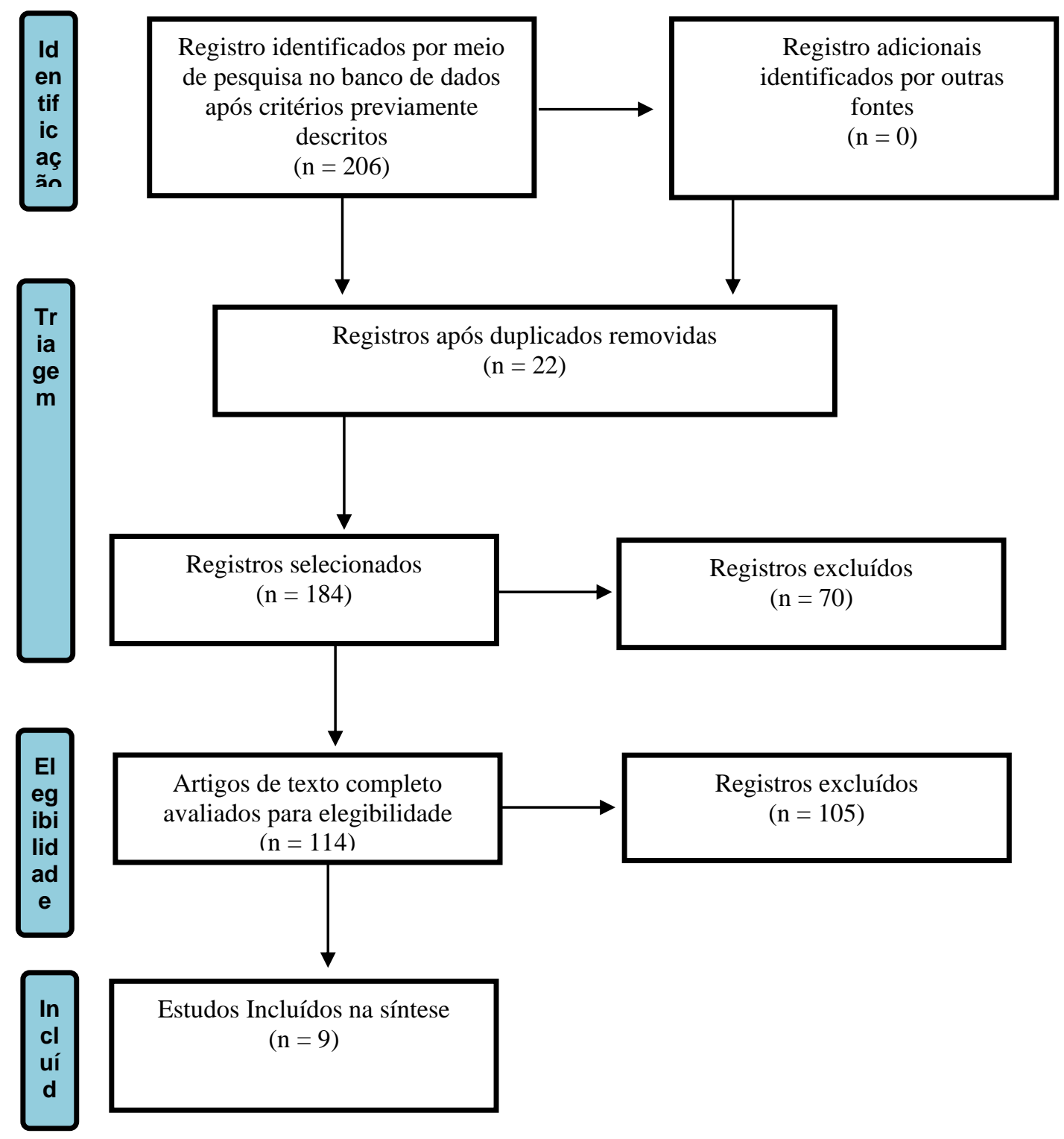

Fonte: Autores.

O Quadro 1 dispõe de 6 artigos da área de obstetrícia e no Quadro 2 são 3 artigos, 1 da área de Urologia e 2 da área de Ginecologia. Dispostos após avaliação criteriosa de acordo com ano de publicação em ordem crescente; objetivo; número de participantes; intervenção e controle; antes e depois da intervenção com o valor de significância e os principais resultados. 
Quadro 1: Síntese dos estudos que abordam o tema “A efetividade da bandagem elástica funcional nas disfunções pélvicas femininas", na área de Obstetrícia.

\begin{tabular}{|c|c|c|c|c|c|c|c|}
\hline $\begin{array}{l}\text { Autor/ } \\
\text { Ano }\end{array}$ & Objetivo & $\mathbf{N}$ & $\begin{array}{l}\text { Intervenção/ } \\
\text { Controle }\end{array}$ & $\begin{array}{c}\text { Localização e formato da } \\
\text { bandagem }\end{array}$ & $\begin{array}{l}\text { Instrumento de } \\
\text { avaliação }\end{array}$ & $\begin{array}{l}\text { Antes/ Depois } \\
\text { Valor de p }\end{array}$ & $\begin{array}{l}\text { Principais } \\
\text { resultados }\end{array}$ \\
\hline $\begin{array}{l}\text { Kaplan, Ş. et } \\
\text { al./ } 2016\end{array}$ & $\begin{array}{l}\text { Determinar a } \\
\text { influência do } \\
\text { Kinesio taping } \\
\text { na dor lombar } \\
\text { relacionada à } \\
\text { gravidez }\end{array}$ & $\begin{array}{c}65 \text { gestantes } \\
\text { Com idade de } 18 \\
\text { e } 40 \text { anos com } 10 \\
\text { e } 30 \text { semanas de } \\
\text { gestação }\end{array}$ & $\begin{array}{l}\text { G KT (33): } \\
\text { Recebeu } 5 \text { dias de } \\
\text { terapia com a } \\
\text { KT } \\
\text { GC (32): Não } \\
\text { recebeu nenhum } \\
\text { tratamento } \\
\text { adicional. } \\
\text { Todas as } \\
\text { participantes } \\
\text { receberam } \\
\text { paracetamol } \\
\text { 1500mg/dia }\end{array}$ & $\begin{array}{l}4 \text { bandas da fita em forma de I } \\
2 \text { bandas: uma de cada lado da } \\
\text { coluna lombar aplicadas } \\
\text { verticalmente da região da CIPI até } \\
\text { a região da } 12^{\text {a }} \text { costela superior, } \\
\text { com técnica de inibição. } \\
2 \text { bandas: fixadas horizontalmente, } \\
\text { com técnica de correção espacial. } \\
\text { Todas as 4: tensão de } 50 \% \text {. }\end{array}$ & $\begin{array}{l}\text { VAS - escala } \\
\text { visual analógica } \\
\text { RMDQ - } \\
\text { Roland-Morris } \\
\text { Disability } \\
\text { Questionnaire }\end{array}$ & $\begin{array}{l}\text { VAS em descanso } \\
\text { GKT X GC }(P< \\
0,001) \\
\text { VAS em } \\
\text { movimento } \\
\text { GKT X GC }(P< \\
0,001) \\
\text { RMDQ } \\
\text { GKT X GC }(P< \\
0,001)\end{array}$ & $\begin{array}{l}\text { O estudo mostrou } \\
\text { que a terapia } \\
\text { combinada com KTe } \\
\text { paracetamol pareceu } \\
\text { ser mais eficaz para } \\
\text { reduzir a dor e } \\
\text { melhorar a } \\
\text { capacidade funcional } \\
\text { no tratamento da dor } \\
\text { lombar relacionada à } \\
\text { gravidez. }\end{array}$ \\
\hline $\begin{array}{c}\text { Cripriano, P.; } \\
\text { Oliveira, } \\
\text { C. } / 2017\end{array}$ & $\begin{array}{l}\text { Verificar a } \\
\text { influência da } \\
\text { bandagem } \\
\text { elástica Kinesio } \\
\text { Tape e da } \\
\text { hidroterapia na } \\
\text { dor pélvica } \\
\text { posterior e na } \\
\text { funcionalidade } \\
\text { das AVDs em } \\
\text { gestantes. }\end{array}$ & $\begin{array}{l}40 \text { gestantes com } \\
\text { idade entre } 18 \text { e } \\
39 \text { anos e idade } \\
\text { gestacional de } 3 \\
\text { ou mais meses. }\end{array}$ & $\begin{array}{c}\text { GE (10): } \\
\text { Hidroterapia e KT } \\
\text { GC (10): } \\
\text { Hidroterapia }\end{array}$ & $\begin{array}{l}\text { A técnica de bloqueio dos } \\
\text { músculos paravertebrais lombares } \\
\text { colocando-se as tiras de KT sobre } \\
\text { esses músculos e combinando-se a } \\
\text { colocação com outra tira na } \\
\text { horizontal, sobre as EIPS, com } \\
\text { tensão de } 25 \% \text { e } 0 \% \text { nas âncoras }\end{array}$ & $\begin{array}{l}\text { Escala Visual } \\
\text { Numérica (EVN) } \\
\\
\text { Questionário de } \\
\text { incapacidade } \\
\text { funcional de } \\
\text { Roland Morris }\end{array}$ & $\begin{array}{l}\text { Média inicial e } \\
\text { reavaliação } \\
\text { EVN } \\
\text { GE }(6.8 ; 4.1 \mathrm{p} \\
=0,001) \\
\text { GC }(5,6 ; 4,2 \\
\text { p=0,003) } \\
\text { GE x GC }(4.1 ; 4.2 \\
\text { p=0.914) } \\
\text { Morris } \\
\text { GE }(9.4 ; 7.6 \\
\text { p=0,011) } \\
\text { GC }(8.8 ; 5.2 \\
\text { p=0,002) } \\
\text { GE x GC }(7.6 ; 5.2 \\
\text { p=0.222) }\end{array}$ & $\begin{array}{l}\text { O trabalho mostrou } \\
\text { que ambos os } \\
\text { tratamentos - } \\
\text { hidroterapia e } \\
\text { bandagem elástica } \\
\text { KT - são eficazes } \\
\text { para o tratamento da } \\
\text { dor pélvica posterior } \\
\text { e da funcionalidade } \\
\text { em gestantes. }\end{array}$ \\
\hline
\end{tabular}




\begin{tabular}{|c|c|c|c|c|c|c|c|}
\hline $\begin{array}{c}\text { Mohamed, E. } \\
\text { A.; El-shamy, } \\
\text { F. F.; Hamed, } \\
\text { H./2017 }\end{array}$ & $\begin{array}{l}\text { Investigar o } \\
\text { efeito da } \\
\text { cinesiotape em } \\
\text { mulheres com } \\
\text { dores nas costas } \\
\text { pós-parto }\end{array}$ & $\begin{array}{l}30 \text { participantes } \\
\text { com idade entre } \\
18 \text { e } 35 \text { anos. } \\
\text { Após e meses } \\
\text { pós-parto. }\end{array}$ & $\begin{array}{l}\quad \text { GE }(15)- \\
\text { Recebeu Kinesio } \\
\quad \text { Tape } \\
\text { GC (15) - Não } \\
\text { recebeu } \\
\text { intervenção }\end{array}$ & $\begin{array}{l}4 \text { fitas em forma de I: } \\
2 \text { bandas: colocadas na vertical } \\
\text { bilateralmente nos músculos } \\
\text { paravertebrais até a região das } \\
\text { nádegas a nível da L1-T12 com } \\
\text { tensão de } 15-25 \% \text { e sem tensão nas } \\
\text { âncoras. } \\
2 \text { bandas: na horizontal no local } \\
\text { mais dolorido com tensão de } 50 \% \\
\text { e sem tensão nas âncoras. }\end{array}$ & $\begin{array}{l}\text { Escala Visual } \\
\text { Analógica } \\
\text { (EVA) } \\
\text { The Back Pain } \\
\text { Function Scale } \\
\text { (BPFS) }\end{array}$ & $\begin{array}{c}\text { Antes da } \\
\text { Intervenção (valor } \\
\text { base) } \\
\text { GKT X GC } \\
\text { VAS - } 0,07 \\
\text { BPFS - 0,34 } \\
\text { Depois da } \\
\text { Intervenção } \\
\text { GKT } \\
\text { VAS - 0,0001 } \\
\text { BPFS - } 0,0001 \\
\text { GC } \\
\text { VAS - } 0,0001 \\
\text { BPFS - } 0,0010\end{array}$ & $\begin{array}{l}\text { O uso de cinesiotape } \\
\text { e exercícios de } \\
\text { correção postural } \\
\text { podem ser usados } \\
\text { como métodos } \\
\text { eficazes e seguros } \\
\text { para o tratamento de } \\
\text { mulheres pós-natais } \\
\text { com dor nas costas e } \\
\text { podem ser } \\
\text { considerados uma } \\
\text { alternativa viável ou } \\
\text { complemento ao } \\
\text { tratamento médico da } \\
\text { dor lombar. }\end{array}$ \\
\hline $\begin{array}{c}\text { Kalinowski, P.; } \\
\text { Krawulska, } \\
\text { A./2017 }\end{array}$ & $\begin{array}{l}\text { Determinar a } \\
\text { influência do } \\
\text { KT na redução } \\
\text { da dor lombar } \\
\text { em mulheres } \\
\text { grávidas. }\end{array}$ & $\begin{array}{l}106 \text { mulheres com } \\
\text { idade entre } 25 \text { e } \\
\text { mais de } 30 \text { anos, } \\
\text { com IG de } 2^{\circ} \text { e } 3^{\circ} \\
\text { trimestre. }\end{array}$ & $\begin{array}{l}\text { Grupo A (53) - } \\
\quad \text { Recebeu o } \\
\text { Kinesio Taping } \\
\quad \text { com tensão. } \\
\text { Grupo B (53) - } \\
\text { Recebeu o } \\
\text { Kinesio Taping } \\
\text { sem tensão. }\end{array}$ & $\begin{array}{l}4 \text { bandas de fita KT em forma de I } \\
\text { Grupo A (Kinesio Taping) - } \\
\text { bilateralmente ao lado da coluna na } \\
\text { posição vertical. Em seguida foi } \\
\text { aplicado mais uma fita na posição } \\
\text { horizontal com tensão de } 75 \% \text { na } \\
\text { região dolorosa. Uma outra fita em } \\
\text { forma de I foi aplicada nos } \\
\text { músculos abdominais para } \\
\text { diminuir a carga da coluna lombar. } \\
\text { Grupo Placebo (Bandagem Polovis } \\
\text { Plus) - Teve os mesmos critérios } \\
\text { de aplicação do KT, porém sem } \\
\text { tensão alguma. }\end{array}$ & $\begin{array}{c}\text { Escala Visual } \\
\text { Analógica } \\
\text { (VAS) } \\
\text { Roland Morris } \\
\text { Disability } \\
\text { Questionnaire } \\
\text { (RMDQ - 2004) }\end{array}$ & $\begin{array}{c}\text { VAS } \\
2 \text { dias após } \\
\text { aplicação } \\
\text { Grupo A (KT) X } \\
\text { Grupo B } \\
\text { (Bandagem } \\
\text { Polovis) }-P \\
<0,0001 \\
7 \text { dias após } \\
\text { aplicação } \\
\text { Grupo A (KT) X } \\
\text { Grupo B } \\
\text { (Bandagem } \\
\text { Polovis) - } P \\
<0,0001 \\
2 \text { e } 7 \text { dias após } \\
\text { aplicação } \\
\text { Grupo A (KT) } \\
P<0,0001 \\
\text { Grupo B }\end{array}$ & $\begin{array}{l}\text { Após a aplicação do } \\
\text { Kinesio taping, VAS } \\
\text { E RMDQ diminuiu } \\
\text { significativamente } \\
\text { em comparação com } \\
\text { o placebo. O efeito } \\
\text { terapêutico começa } \\
\text { no dia } 2 \text { e é mantido } \\
\text { por } 2 \text { dias após a } \\
\text { remoção da fita. }\end{array}$ \\
\hline
\end{tabular}


Research, Society and Development, v. 10, n. 11, e215101119349, 2021

(CC BY 4.0) | ISSN 2525-3409 | DOI: http://dx.doi.org/10.33448/rsd-v10i11.19349

\begin{tabular}{|c|c|c|c|c|c|c|c|}
\hline & & & & & & $\begin{array}{c}\text { (Bandagem } \\
\text { Polovis) } \\
\\
P<0,8087 \\
\text { RMDQ } \\
\text { Grupo A (KT) X } \\
\text { Grupo B } \\
\text { (Bandagem } \\
\text { Polovis) } \\
P<0,0001\end{array}$ & \\
\hline $\begin{array}{l}\text { Miquelutti, M. } \\
\text { A.; Silveira, } \\
\text { C.; Cecatti, J. } \\
\text { G./2018 }\end{array}$ & $\begin{array}{c}\text { A eficácia e } \\
\text { segurança do } \\
\text { uso de KT } \\
\text { durante o } \\
\text { trabalho de parto } \\
\text { em relação à } \\
\text { dor, satisfação } \\
\text { materna e } \\
\text { resultados } \\
\text { obstétricos e } \\
\text { neonatais. }\end{array}$ & $\begin{array}{c}60 \text { gestantes com } \\
\text { idade gestacional } \\
\text { entre } 37 \text { e } 42 \text { duas } \\
\text { semanas }\end{array}$ & $\begin{array}{c}\text { Grupo Kinesio } \\
\text { Taping (30) - } \\
\text { Recebeu a } \\
\text { bandagem com } \\
\text { tensão. } \\
\text { Grupo Controle } \\
\text { (30) - Recebeu } \\
\text { bandagem sem } \\
\text { tensão. } \\
\text { Grupo KT }-\mathrm{N}=29 \\
\text { Grupo } \mathrm{C}-\mathrm{N}=27\end{array}$ & $\begin{array}{l}\text { Em ambos os grupos, foi usado um } \\
\text { PerformTex TM em forma de I } \\
\text { Grupo KT } \\
2 \text { bandas: aplicadas bilateralmente } \\
\text { na região paravertebral entre os } \\
\text { nervos espinhais T10 e L1 com } \\
\text { tensão de } 10-25 \% \text {. } \\
4 \text { bandas: aplicadas formando uma } \\
\text { estrela na região do sacro entre os } \\
\text { nervos espinhais S2 e S4 com } \\
\text { tensão de } 10-25 \% \text {. } \\
\text { Grupo Controle } \\
1 \text { banda: aplicada verticalmente na } \\
\text { região torácica superior, na } \\
\text { localização dos nervos espinhais } \\
\text { T1-T4, sem tensão. }\end{array}$ & $\begin{array}{l}\text { Escalas VAS e } \\
\text { Present Pain } \\
\text { Intensity (PPI) } \\
\text { (McGill } \\
\text { abreviada) } \\
\text { Escores de } \\
\text { Apgar do } \\
\text { primeiro e quinto } \\
\text { minutos de vida } \\
\text { Escala do tipo } \\
\text { Likert de } 5 \\
\text { pontos }\end{array}$ & $\begin{array}{c}\text { VAS (primeira } \\
\text { hora) } \\
\text { Grupo } \mathrm{KT}-\mathrm{P}= \\
0.32 \\
\text { Grupo } \mathrm{C}-P= \\
0,002\end{array}$ & $\begin{array}{c}\text { A bandagem } \\
\text { cinesiológica não foi } \\
\text { clinicamente ou } \\
\text { estatisticamente } \\
\text { eficaz no controle da } \\
\text { dor do parto, e não } \\
\text { foi associada com os } \\
\text { resultados obstétricos } \\
\text { restantes avaliados. } \\
\text { No entanto, não } \\
\text { interferiu na } \\
\text { vitalidade fetal e foi } \\
\text { bem aceito pelos } \\
\text { participantes do } \\
\text { estudo }\end{array}$ \\
\hline
\end{tabular}


Research, Society and Development, v. 10, n. 11, e215101119349, 2021

(CC BY 4.0) | ISSN 2525-3409 | DOI: http://dx.doi.org/10.33448/rsd-v10i11.19349

\begin{tabular}{|c|c|c|c|c|c|c|c|}
\hline $\begin{array}{l}\text { Chamnankrom, } \\
\text { M. et al//2020 }\end{array}$ & $\begin{array}{l}\text { Comparar o } \\
\text { efeito da fita } \\
\text { elástica com a } \\
\text { fita elástica } \\
\text { (placebo) no } \\
\text { tratamento da } \\
\text { dor lombar em } \\
\text { gestantes }\end{array}$ & $\begin{array}{c}40 \text { gestantes } \\
\text { Idade entre } 18 \text { e } \\
\quad 40 \text { anos } \\
\quad \text { Período } \\
\text { gestacional entre } \\
28 \text { e } 38 \text { semanas }\end{array}$ & $\begin{array}{l}\text { Grupo Elástico } \\
\quad(20) \text { - Foi } \\
\text { aplicado a fita } \\
\text { Kinesio taping } \\
\text { com tensão de } \\
\quad 50 \% \text {. } \\
\text { Grupo Controle } \\
\text { (20) - Foi } \\
\text { aplicado a fita, } \\
\text { porém não teve } \\
\text { tensão. }\end{array}$ & $\begin{array}{c}\text { Grupo Elástico } \\
4 \text { fitas elásticas } 5 \text { x0,5 cm em forma } \\
\text { de I na região lombar. } \\
2 \text { bandas: aplicada verticalmente } \\
\text { da SI a } 12^{\mathrm{a}} \text { costela bilateralmente, } \\
\text { com tensão de } 50 \% \text {. } \\
2 \text { bandas: na horizontal começando } \\
\text { no processo espinhoso até a linha } \\
\text { coronal da } 4^{\mathrm{a}} \text { e } 5^{\mathrm{a}} \text { coluna lombar a } \\
\text { nível da última costela, } \\
\text { bilateralmente, tensão de } 50 \% \text {. } \\
\text { Grupo Placebo } \\
\text { Receberam as mesmas aplicações } \\
\text { da fita na mesma região, sem } \\
\text { tensão. }\end{array}$ & $\begin{array}{l}\text { Escala Visual } \\
\text { Analógica } \\
\text { (EVA) } \\
\text { Physiotherapy } \\
\text { Evidence } \\
\text { Database (PE- } \\
\text { Dro) para } \\
\text { qualidade } \\
\text { metodológica } \\
\text { Roland-Morris } \\
\text { Disability } \\
\text { Questionnaire } \\
\text { (RMDQ) } \\
\text { Aplicativo } \\
\text { Android } \\
\text { PostureScreen } \\
\text { Mobile }\end{array}$ & $\begin{array}{c}\text { VAS } \\
\text { Pós intervenção } \\
\text { imediata (dor) } \\
\text { GE }-P=0,003 \\
\text { GP }-P=0,06 \\
\text { Uma semana após } \\
\text { aplicação (dor) } \\
\text { GE }-P<0,001 \\
\text { GP }-P=0,04 \\
\text { GEXGP = } \\
P<0,001 \\
\text { Uma semana após a } \\
\text { aplicação } \\
\text { (incapacidade) } \\
\text { GExGP = } \\
P<0,05 \\
\text { Uma semana após a } \\
\text { aplicação } \\
\text { (velocidade de } \\
\text { caminhada) } \\
\text { GEXGP = } \\
P<0,05\end{array}$ & $\begin{array}{l}\text { O grupo elástico } \\
\text { apresentou um efeito } \\
\text { positivo na redução } \\
\text { da dor lombar após } \\
\text { uma semana com } \\
\text { uma melhora de } 95 \% \\
\text { em relação ao grupo } \\
\text { placebo e melhora na } \\
\text { função física em } \\
\text { mulheres grávidas no } \\
\text { terceiro trimestre. }\end{array}$ \\
\hline
\end{tabular}

EVA: Escala Visual Analógica; RMDQ: Roland-Morris Disability Questionnaire KT: Kinesio Taping; AVDs: Atividades de Vida Diárias; EVN: Escala Visual Numérica. Fonte: Autores. 
Quadro 2: Síntese dos estudos que abordam o tema "A efetividade da bandagem elástica funcional nas disfunções pélvicas femininas", nas áreas de Ginecologia e Urologia.

\begin{tabular}{|c|c|c|c|c|c|c|c|}
\hline $\begin{array}{l}\text { Hasenack. et } \\
\text { al./2016 }\end{array}$ & $\begin{array}{l}\text { Avaliar os efeitos } \\
\text { da bandagem } \\
\text { funcional sobre a } \\
\text { dor e AVDs de } \\
\text { estudantes } \\
\text { universitárias com } \\
\text { DP. }\end{array}$ & $\begin{array}{l}22 \text { estudantes } \\
\text { universitárias } \\
18 \text { a } 35 \text { anos }\end{array}$ & $\begin{array}{l}\text { GA (11): Recebeu } \\
\text { no primeiro mês } \\
\text { intervenção com } \\
\text { BEF e no segundo } \\
\text { mês não houve } \\
\text { intervenção } \\
\text { GB (11): No } \\
\text { primeiro mês não } \\
\text { recebeu } \\
\text { intervenção com a } \\
\text { BEF e no segundo } \\
\text { mês recebeu } \\
\text { intervenção }\end{array}$ & $\begin{array}{l}\text { Região } \\
\text { lombossacra, de } \\
\text { L5 a S1(com } \\
\text { tensão de 100\% no } \\
\text { centro na região } \\
\text { central da } \\
\text { bandagem) e } \\
\text { outras duas tiras } \\
\text { em formato de } \\
\text { cruz na região } \\
\text { abdominal inferior } \\
\text { (com tensão de } \\
\text { 100\% no centro na } \\
\text { região central da } \\
\text { bandagem e sem } \\
\text { tensão nas } \\
\text { extremidades) }\end{array}$ & $\begin{array}{l}\text { Escala Visual } \\
\text { Analógica (EVA), } \\
\text { Questionário de } \\
\text { Avaliação e o } \\
\text { Mapa Corporal }\end{array}$ & $\begin{array}{l}\text { Intensidade da dor } \\
\text { A: } N S \\
B: 3^{\circ} \text { dia ( } p= \\
0,01), 4^{\circ} \text { dia ( } p= \\
0,02) \text { e média geral } \\
(\mathrm{p}=0,04) \text {, a favor } \\
\text { da intervenção. } \\
\text { Número de dias } \\
\text { A: } 1,9 \text { e } 2,9 \\
(\mathrm{P}=0,02) \\
\mathrm{B}: 2,6 \text { e } 1,8 \\
(\mathrm{P}=0,04)\end{array}$ & $\begin{array}{l}\text { A bandagem é } \\
\text { benéfica na } \\
\text { dismenorreia } \\
\text { primária em } \\
\text { universitárias, com } \\
\text { efeitos positivos } \\
\text { sobre a redução da } \\
\text { intensidade e a } \\
\text { duração da dor. }\end{array}$ \\
\hline $\begin{array}{l}\text { Souza et al./ } \\
2019\end{array}$ & $\begin{array}{l}\text { Verificar os efeitos } \\
\text { do biofeedback, da } \\
\text { bandagem elástica } \\
\text { funcional e da } \\
\text { associação de } \\
\text { ambas } \\
\text { as técnicas na QV } \\
\text { de mulheres com } \\
\text { IUE na pós } \\
\text { menopausa. }\end{array}$ & $\begin{array}{l}12 \text { mulheres com } \\
\text { idade entre } 43 \text { e } 68 \\
\text { anos. } \\
\text { (média } 56,16 \pm \\
6,83 \text { ) }\end{array}$ & $\begin{array}{l}\text { Grupo A (4): } \\
\text { submetido ao BP. } \\
\text { Grupo B (4): } \\
\text { submetido à } \\
\text { aplicação de BEF. } \\
\text { Grupo C (4): } \\
\text { submetido ao BP } \\
\text { associado à } \\
\text { aplicação de BEF. }\end{array}$ & $\begin{array}{l}\text { A BEF foi aplicada } \\
\text { na região das } \\
\text { vértebras sacrais } \\
\text { S2 }-S 4, \text { com } \\
\text { medidas } 5 \times 5 \mathrm{~cm} \text { na } \\
\text { região de forma } \\
\text { horizontal, da } \\
\text { esquerda para a } \\
\text { direita, sendo que } \\
\text { nenhuma tensão } \\
\text { foi dada nas } \\
\text { extremidades e no } \\
\text { centro da fita foi } \\
\text { dada tensão } \\
\text { máxima. }\end{array}$ & $\begin{array}{c}\text { ICIQ-SF } \\
\text { e } \\
\text { I-QOL }\end{array}$ & $\begin{array}{l}\text { ICIQ-SF / I-QOL } \\
\text { Grupo A: } \\
\text { DP antes: } \\
\text { 14,45/52,95 } \\
\text { depois:2,25/92,95 } \\
2 \text { meses após o } \\
\text { tratamento: 4/ } \\
81,29 p \leq 0,05 \\
\text { Grupo B: } \\
\text { DP antes: } 14,75 / \\
74,76 \\
\text { depois:6,5/88,17 } \\
2 \text { meses após o } \\
\text { tratamento:6,5/ } \\
87,17 \\
\text { p } \leq 0,05 \\
\text { Grupo C: } \\
\text { DP antes:19/ 50,90 }\end{array}$ & $\begin{array}{l}\text { O Grupo A e B } \\
\text { apresentou } \\
\text { melhora na } \\
\text { qualidade de vida, } \\
\text { o último mantendo } \\
\text { um resultado } \\
\text { melhor após um } \\
\text { mês de término do } \\
\text { tratamento. O } \\
\text { Grupo C resultou } \\
\text { em uma melhora } \\
\text { da QV nos três } \\
\text { períodos. }\end{array}$ \\
\hline
\end{tabular}


Research, Society and Development, v. 10, n. 11, e215101119349, 2021

(CC BY 4.0) | ISSN 2525-3409 | DOI: http://dx.doi.org/10.33448/rsd-v10i11.19349

\begin{tabular}{|c|c|c|c|c|c|c|c|}
\hline & & & & & & $\begin{array}{l}\text { depois: } 10 / 78,40 \\
2 \text { meses após o } \\
\text { tratamento: } \\
7,5 / 81,36 \\
\mathrm{p} \leq 0,05\end{array}$ & \\
\hline $\begin{array}{l}\text { Celenay., et } \\
\text { al. } / 2020\end{array}$ & $\begin{array}{l}\text { Investigar os } \\
\text { efeitos da kinesio } \\
\text { Taping na dor, } \\
\text { ansiedade e } \\
\text { queixas menstruais } \\
\text { em mulheres com } \\
\text { Dismenorreia } \\
\text { primária e } \\
\text { comparar esta } \\
\text { aplicação com o } \\
\text { grupo controle e o } \\
\text { grupo de fita } \\
\text { simulada. }\end{array}$ & $\begin{array}{l}45 \text { mulheres } \\
18 \text { a } 35 \text { anos }\end{array}$ & $\begin{array}{l}\text { G1: Aplicação da } \\
\text { BEF } \\
\text { G2: Aplicação da } \\
\text { BEF } \\
\text { G3: Nenhuma } \\
\text { aplicação de } \\
\text { bandagem elástica } \\
\text { funcional ou } \\
\text { intervenção. }\end{array}$ & $\begin{array}{l}\text { G1:Em formato de } \\
\text { cruz na região } \\
\text { suprapúbica e na } \\
\text { região sacral (S1), } \\
\text { com a técnica } \\
\text { ligamentar ( } 75 \% \text { a } \\
\text { 100\% de } \\
\text { estiramento). } \\
\text { G2:Em formato de } \\
\text { cruz, no trocante } \\
\text { maior direito do } \\
\text { fêmur, sem tensão } \\
\text { e sem técnica } \\
\text { ligamentar. }\end{array}$ & $\begin{array}{l}\text { Escala Visual } \\
\text { Analógica (EVA) } \\
\text { e o questionário } \\
\text { State-Trait Anxiety } \\
\text { Inventory }\end{array}$ & $\begin{array}{l}\text { Mediana } \\
\text { EVA antes } \\
\text { G1XG2XG3 (P = } \\
0.088) \\
\text { EVA depois } \\
\text { G1XG2XG3(P < } \\
0.001) \\
\text { State-Trait Anxiety } \\
\text { Inventory antes } \\
\text { G1XG2XG3 (P = } \\
\text { 0.565) } \\
\text { State-Trait Anxiety } \\
\text { Inventory depois } \\
\text { G1XG2XG3 (P < } \\
\text { 0.001) }\end{array}$ & $\begin{array}{l}\text { A diminuição dos } \\
\text { níveis dor, } \\
\text { ansiedade e } \\
\text { algumas queixas } \\
\text { menstruais após a } \\
\text { aplicação foram } \\
\text { maiores no grupo } 1 \\
\text { do que nos outros } \\
\text { dois grupos. } \\
\text { Demonstrando } \\
\text { assim, que a } \\
\text { aplicação da } \\
\text { kinesio taping é } \\
\text { um método eficaz } \\
\text { em mulheres com } \\
\text { dismenorreia } \\
\text { primária. }\end{array}$ \\
\hline
\end{tabular}

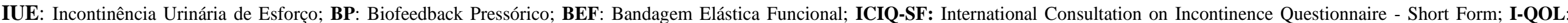

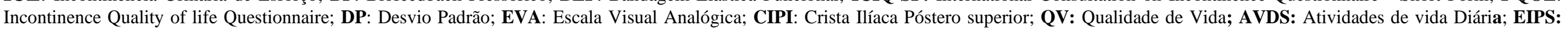
Espinha Ilíaca Póstero Superior. Fonte: Autores. 
O processo de buscas por artigos foi dividido em três áreas: Obstetrícia, ginecologia e Uroginecologia. Dentre os artigos publicados, a área mais requerida foi à obstetrícia (Figura 3). Isso acontece provavelmente, porque nos últimos anos observou-se um aumento de estudos envolvendo a fisioterapia obstétrica.

Figura 3: Divisão dos artigos por área: Obstetrícia, Ginecologia e Urologia.

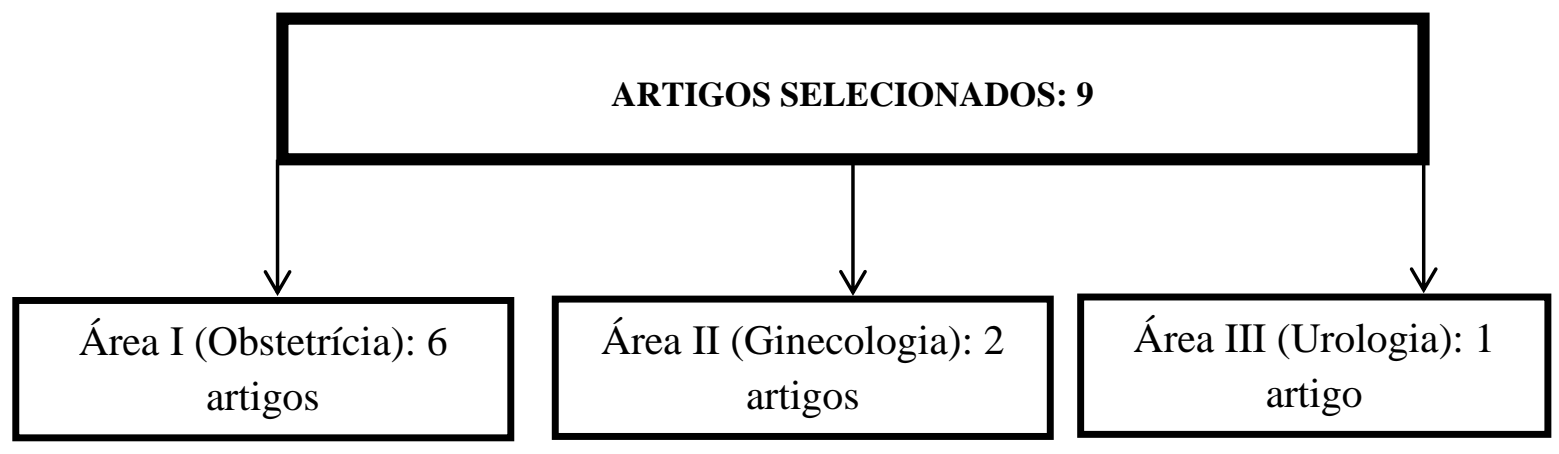

Fonte: Autores.

As disfunções pélvicas, mesmo que temporárias, são limitantes e afetam aspectos sociais e psicológicos, com importante comprometimento na qualidade de vida. Além disso, alteram condições de saúde física, atividades do cotidiano, o bem-estar emocional e a vida familiar e social (Berghmans et al, 2015). Rodrigues et al. (2011), concluíram em seu estudo que $78,3 \%$ das mulheres apresentavam quadro de ansiedade/depressão associada às DAPs.

O tratamento das DAPs geralmente ultrapassa barreiras emocionais, psicológicas e culturais que permeiam o universo feminino, principalmente devido ao contexto histórico ao qual a nossa sociedade está inserida, isso gera demora ao buscar um tratamento específico e dificuldade na continuidade dele (Kiebzak et al., 2012). Terapias complementares não farmacológicas podem ser utilizadas como nova forma de tratamento das DAPS. Através de técnicas como a bandagem elástica, que é um método não invasivo e sem efeitos adversos, pode-se promover a estimulação do sistema tegumentar através dos receptores somatossensoriais, promovendo alívio dos sintomas associados às DAPs (Yuejie et al., 2019).

Todos os artigos incluídos neste estudo são recentes, publicados nos últimos 5 anos, o que mostra que a Bandagem Elástica vem sendo cada vez mais utilizada como recurso terapêutico no tratamento das DAPs. Após análise dos artigos, observou-se que dos 9 selecionados, 8 utilizaram a Escala Visual Analógica (EVA) para avaliar a intensidade da dor. Em um estudo realizado por Jensen (2003) afirmou-se que as escalas de itens simples como a EVA, são adequadas para conceitos unidimensionais, como a dor e que podem ser aplicadas rapidamente, delimitando respostas rápidas (Jensen et al., 2003).

Como já referido, a área onde encontrou-se o maior número de publicações de acordo com os critérios estabelecidos no processo de buscas por artigo, foi a obstetrícia. Antes de ser discutida a análise individual desses estudos, podemos ressaltar o que há de concordância entre a relação da forma de aplicação, o corte da fita, posicionamento, tempo de uso, tensão e localização da bandagem (Artioli et al., 2014). Todos os artigos utilizaram o corte em forma de "I" que é um dos principais tipos de cortes para a realização de técnicas como a de inibição e toda ancoragem, inicial ou distal sempre possuindo 0\% de tensão, que é conhecida como normotensão. O local ou tecido no qual é aplicada a tensão é chamada de zona terapêutica (Kase et al., 2013).

A lombalgia é uma queixa comum na gravidez, devido às mudanças que o organismo sofre para acomodar o feto. Alterações musculoesqueléticas como o aumento da curva torácica e lombar, propiciam o surgimento da dor lombar (Lima \& Antônio, 2009). Nos estudos encontrados dois analisaram a dor e funcionalidade. No estudo de Mohamed et al. (2018), foram estudadas 30 gestantes $(\mathrm{GE}=15$ e $\mathrm{GC}=15)$ onde o $\mathrm{GE}$ teve a aplicação da $\mathrm{KT}$ associado à exercícios e orientações sobre dor 
lombar, e os resultados foram positivos mais que no GC. Além disso, é importante ressaltar que o método pode ser utilizado de forma segura, porque ele não apresentou efeitos colaterais, tem menor custo e é de fácil aplicação.

No estudo de Cipriano e Oliveira (2017), buscaram verificar também a influência da KT para o alívio da dor e da funcionalidade de 20 gestantes ( $\mathrm{GE}=10$ e GC $=10)$, e os resultados se mostraram bastante satisfatórios em relação a redução da dor e a melhora da funcionalidade, reafirmando que a aplicação da KT associada ou não com algum outro tratamento, melhora de forma positiva o alívio da dor e da funcionalidade.

Kase et al. (2013), relatam que o simples contato das bandagens, associado ao deslizamento, a pressão e a tensão da pele permitem o alívio da dor e a sensação de desconforto local e em tecidos subjacentes. Afirma ainda, que o KT pode desencadear efeitos diretamente sobre a musculatura, estimulando e ativando o músculo ou um grupo muscular durante o movimento, quando se aplica uma tração sobre a pele você pode imediatamente otimizar a comunicação neural com os mecanorreceptores, aumentando assim o número de unidades motoras recrutadas durante a contração. Corroborando com os resultados encontrados no estudo de Chamnankrom et al. (2021), onde ele avaliou 40 gestantes ( $\mathrm{GE}=20$ e $\mathrm{GC}=20$ ) com dor lombar devido a gravidez e obteve resultados importantes em relação a funcionalidade, pois após a aplicação da fita o grupo experimental apresentou aumento significativo em relação a velocidade de caminhada, em relação ao grupo controle.

Em grande parte das aplicações, são utilizadas tensões abaixo de 50\% que desencadeiam efeitos sensitivos, já em tensões de 50\% ou mais causam maiores efeitos mecânicos (Kase et al., 2013). Comprovado no estudo de Kaplan et al. (2016), que realizou um estudo com 71 gestantes $(\mathrm{KT}=36$ e GC $=35)$, eles avaliaram o efeito da $\mathrm{KT}$ na dor e na incapacidade funcional, onde foi usado a bandagem com $50 \%$ de tensão associado com o uso do paracetamol (Parol $®, 1500 \mathrm{mg} / \mathrm{dia}$ ) por 5 dias, e obtiveram resultados satisfatórios tanto na dor quanto na incapacidade funcional em relação ao grupo controle $(\mathrm{P}<$ $0,001)$.

Nos estudos de Mohamed et al. (2018); Miquelutti et al. (2019); Cipriano \& Oliveira, (2017); Kalinowski \& Krawulska, (2017), foi utilizada a técnica de inibição com tensão de $10 \%$ a $25 \%$. Técnica essa que reduz músculos excessivamente fortes, ativos, hipertônicos, comuns em condições agudas como espasmos musculares que geram dores, objetivaram a redução de dores nas gestantes e obtiveram resultados semelhantes na melhora da dor (Kase et al., 2013).

Em outros dois trabalhos Chamnankrom et al. (2021) e Kaplan et al. (2016), usaram também a técnica de inibição, porém utilizaram a tensão de 50\% na zona terapêutica. Conforme Kase et al. (2013), a tensão durante a aplicação da Kinesio taping é classificada como máxima - 75\% a 100\%; severa ou rígida - 50\% a 75\%; moderada - 50\%; leve - $25 \%$ a $35 \%$ e; muito leve $-10 \%$ a 15\%. Assim, quanto menor a tensão, mais efeitos sensitivos são desencadeados; quanto maior, mais efeitos mecânicos.

De acordo com os critérios estabelecidos na busca pelos artigos, dentro da área da ginecologia, foram encontrados apenas artigos que exploraram os benefícios da bandagem elástica em mulheres com dismenorreia primária (DP). Isso acontece provavelmente, porque a DP é o problema ginecológico mais comum entre mulheres em idade reprodutiva e a maior causa de restrição de suas atividades, desta forma, estão sendo realizados muitos artigos envolvendo esta patologia e novas formas de tratamento. O que justifica a necessidade de se estudar de forma mais aprofundada novas formas de alívio da dor e melhora da qualidade de vida dessas mulheres (Kelle et al., 2016).

Ao analisar os artigos selecionados, notou-se que a média de idade das mulheres participantes era de 18 a 35 anos. De acordo com Diegolin \& Diegolin, (2006), a DP possui maior prevalência em adolescentes e jovens mulheres, onde conforme a etiologia se tem maior liberação de prostaglandinas no fluxo menstrual, principalmente da prostaglandina F2 (PGF2).

A dismenorreia é definida como uma cólica menstrual que ocorre imediatamente antes ou durante a menstruação, na ausência de qualquer patologia pélvica orgânica, e pode ser acompanhada por algumas queixas, como dor lombar, fadiga, 
náusea, vômito, diarreia e insônia (Iacovides et al., 2015). Segundo Brown \& Brown, (2010), 80\% das mulheres apresentam, náuseas e vômitos e 50\% diarreia, como sintomas associados à DP. Acredita-se que a bandagem elástica solicita normalização da função muscular, por meio da inibição ou facilitação da contração, corrigindo o posicionamento articular, melhora o retorno venoso e linfático, gerando diminuição do edema local e redução da dor (Kaur et al., 2017).

Uma pesquisa realizada por Souza \& Mejia, (2014), com 26 voluntárias portadoras de DP, foi aplicada a Escala Visual Analógica (EVA) antes e após a aplicação da bandagem, para avaliar a dor das voluntárias. Todas as voluntárias usaram a bandagem por 3 dias, e relataram que a dor diminuiu a partir do segundo dia de uso, confirmando a eficácia da bandagem elástica na redução da dor.

A dor é uma das principais causas que levam as pessoas a incapacitar-se de seus afazeres, gerando graves consequências para as mulheres com dismenorreia primária. Rodrigues et al. (2011), comprovaram em seu estudo que em $65,7 \%$ das mulheres apresentavam limitações das atividades diárias, associadas a DP. Nesse sentido, um estudo foi realizado para avaliar os efeitos da bandagem elástica funcional (BEF) sobre a dor e as atividades de vida diária (AVD's) de estudantes universitárias com DP. O ensaio clínico foi realizado com 22 mulheres, divididas em dois grupos, A e B. No primeiro mês, o grupo A recebeu intervenção com a BEF e no grupo B fez-se apenas o monitoramento dos sintomas. No mês subsequente, foram invertidos os procedimentos entre os grupos. Os resultados do estudo demonstraram que houve redução significativa na intensidade da dor e do número de dias com DP, em ambos os grupos, quando comparados os momentos de controle e intervenção dos dois grupos. Isso sugere que a bandagem elástica possui efeitos benéficos na DP, com redução da duração, intensidade da dor e redução da interferência da DP nas AVD. Isso sugere que essa proposta, não invasiva e com poucos efeitos colaterais, deve ser considerada no tratamento da dismenorreia primária (Hasenack et al., 2016).

Outros autores corroboram com os efeitos benéficos da bandagem elástica funcional na redução da dor, possivelmente associada à melhora da circulação local e depuração das substâncias inflamatórias. Onde avaliaram e comprovaram os efeitos de uma intervenção com bandagem elástica para reduzir a dor em mulheres com DP (GarzonRodriguez, 2013). Em pesquisa mais recente, Celenay et al. (2020), compararam os efeitos da aplicação da bandagem elástica (BE) na dor, ansiedade e queixas menstruais em mulheres com dismenorreia primária. As participantes foram divididas em 3 grupos, onde o primeiro grupo recebeu intervenção com a BE com a técnica ligamentar, o segundo grupo não recebeu nenhuma intervenção com a BE e o terceiro grupo recebeu intervenção com a BE, porém sem nenhuma técnica ligamentar. Para avaliação dos níveis dor e ansiedade, foram utilizados antes e após a aplicação da bandagem, a EVA e o questionário State Anxiety Inventory, respectivamente. Os achados do estudo mostraram que a diminuição da dor, níveis de ansiedade e de algumas queixas menstruais foram maiores no grupo que recebeu a intervenção com BE com a técnica ligamentar, em comparação com os outros dois grupos, que não apresentaram melhora estaticamente significativa dos níveis de dor e ansiedade. Os resultados obtidos no grupo que recebeu intervenção com a BE, podem ter se originado do mecanismo de ação reflexivo envolvendo pele-órgão e aumento do fluxo sanguíneo. Isso sugere que a bandagem elástica pode ser usada para reduzir os sintomas da DP e que pode ser um método complementar na redução da ansiedade e algumas queixas menstruais que acompanham a dor menstrual.

Dentro da área da urologia de acordo com os critérios estabelecidos na busca pelos artigos, foi encontrado apenas um artigo que explorou os benefícios da bandagem elástica em mulheres com incontinência urinária de esforço (IUE). A incontinência urinária é uma das queixas mais frequentes entre mulheres em todas as faixas etárias, sendo mais alta a partir da terceira década de vida (Souza et al., 2017). Os autores consultados concordam que a IUE é o tipo mais comum de incontinência urinária na mulher. Para Carvalho et al. (2014), a IUE é de causa multifatorial, fatores como cirurgia, hipoestrogenismo, multiparidade, alterações pélvicas e avanço da idade, colaboram para o não fechamento uretral. 
Segundo Souza et al. (2019), em sua pesquisa com 12 voluntárias, a incidência e a prevalência da incontinência urinária variam de acordo com a faixa etária e a população estudada, o que condiz com a média de idade do artigo analisado que foi $56,16 \pm 6,83$ anos. Elas apresentaram características predisponentes para a incontinência urinária, tais como: IMC elevado, tempo de amenorreia e perda de urina acima de 5 anos, $90 \%$ haviam realizado algum tipo de cirurgia ginecológica e prevalência de parto por via vaginal.

A pesquisa foi dividida em 3 grupos, sendo o GA (apenas com o biofeedback), GB (apenas BEF), GC (Biofeedback associado com BEF). Desse modo, evidenciando os resultados obtidos quando comparados os tratamentos, verificou-se que o GA e o GB apresentaram melhora na qualidade de vida, porém, o segundo manteve um resultado melhor após um mês do término do tratamento e o GC resultou em uma melhora da qualidade de vida nos três períodos. Sugerindo assim, que a BEF é uma técnica eficaz no tratamento da incontinência urinária (Souza et al., 2019).

Confirmando os resultados de Freire et al. (2016), que observaram em seu estudo a eficácia da BFE na redução da perda de urina e na melhora na qualidade de vida, porém, neste estudo, foram tratados outros tipos de IU e não somente a de esforço. De acordo com estes autores, a BFE deve ser empregada como adjuvante de outros procedimentos no tratamento da IU, com o intuito de potencializar os efeitos e a durabilidade da técnica. Através da análise dos artigos, foi possível compreender a eficácia da Bandagem elástica funcional no tratamento das disfunções pélvicas femininas. Principalmente na tentativa de reduzir a intensidade e duração dos sintomas, associados às DAPs.

\section{Conclusão}

Diante dos resultados obtidos nesta revisão, foi possível confirmar que Bandagem Elástica Funcional atua de maneira eficaz no tratamento das disfunções pélvicas femininas, promovendo bons resultados clínicos em relação dor, ansiedade, qualidade de vida e outros sintomas associados às DAPs. Os ensaios clínicos utilizando a Bandagem Elástica Funcional em mulheres com DAPS ainda são escassos e, portanto, mais estudos de alta qualidade metodológica devem ser realizados investigando a eficácia da Bandagem Elástica Funcional nas Disfunções Pélvicas Femininas, para uma melhor evidência científica.

Para estudos futuros, sugere-se a realização de ensaios clínicos randomizados e controlados, onde sejam investigados e descritos as formas de aplicação da BEF, seus possíveis efeitos adversos e que seja mais especificado os possíveis benefícios da BEF nas disfunções pélvicas femininas para os desfechos dor, ansiedade e qualidade de vida. Através desses dados, a utilização da Bandagem Elástica Funcional terá um maior respaldo científico e poderá ser reproduzida de forma mais técnica no tratamento das Disfunções Pélvicas Femininas.

\section{Referências}

Ali, B., Al-Wabel, N. A., Shams, S., Ahamad, A., Khan, S. A., \& Anwar, F. (2015). Essential oils used in aromatherapy: A systemic review. Asian Pacific Journal of Tropical Biomedicine, 5 (8), 601-611.

Amaral, F., \& Silveira, C. (2018). Manual técnico: cuidados naturais da cabeça aos pés. Brasilia: Wnf.

Andrei, P., \& Del Comune, A. P. (2005). Aromaterapia e suas aplicações. Centro Universitário São Camilo, 11 (4), $57-68$.

Araújo, L. M. D., Silva, J. M. N. D., Bastos, W. T., \& Ventura, P. L. (2012). Diminuição da dor em mulheres com dismenorreia primária, tratadas pelo método Pilates. Revista Dor, 13, 119-123.

Bakhtshirin, F., Abedi, S., YusefiZoj, P., \& Razmjooee, D. (2015). The effect of aromatherapy massage with lavender oil on severity of primary dysmenorrhea in Arsanjan students. Iranian journal of nursing and midwifery research, 20 (1), 156.

Baudoux, D. (2018). O Grande Manual de Aromaterapia de Dominique Baudoux. Trad.: Mayra Corrêa e Castro. Belo Horizonte. Editora Lazslo.

Benitez, L. B., da Silva, C. D. M., \& da Costa Alvares, L. (2016). Utilização da hortelã-pimenta como agente no controle de infecções relacionadas à assistência à saúde (IRAS). Scientia Plena, 12 (12). 
Brasil. Ministério da Saúde. Departamento de Ciência e Tecnologia (2012). Diretrizes metodológicas: elaboração de revisão sistemática e metanálise de ensaios clínicos randomizados [Internet]. Brasília(DF): Editora do Ministério da Saúde.

Brasil. Ministério da Saúde (BR). (2018). Portaria 702, de 21 de março de 2018. Altera a Portaria de Consolidação nº 2/GM/MS, de 28 de setembro de 2017, para incluir novas práticas na Política Nacional de Práticas Integrativas e Complementares-PNPIC.

Cassar, M. P. (2001). Manual de massagem terapêutica. Barueri: Editora Manole.

Clarke, M., \& Chalmers, I. (2018). Reflections on the history of systematic reviews. BMJ Evidence Based Medicine, n. 23, $121-122$.

Costa, I. B., Dantas, D. V., Dantas, R. A. N., Oliveira, S. P., Aiquoc, K. M., \& Sarmento, S. D. G. (2019). Terapias não farmacológicas para o manejo da dor em vítima de queimaduras: uma revisão sistemática. Revista Inspirar: movimento e saúde, 19 (2), 1-20.

Da Cunha, A. P., Nogueira, M. T., \& Roque, O. R. (2012). Plantas aromáticas e óleos essenciais: composição e aplicações. Fundação Calouste Gulbenkian.

Da Silva, I. T. S., de Araújo, A. C., de Medeiros, Y. E., da Costa Santos, R. S., Góis, M. M. D. C. D., \& da Silva, R. A. R. (2020). O uso da aromaterapia no contexto da enfermagem: uma revisão integrativa. Revista Eletrônica de Enfermagem, 22.

Daniel, A. N., Sartoretto, S. M., Schmidt, G., Caparroz-Assef, S. M., Bersani-Amado, C. A., \& Cuman, R. K. N. (2009). Anti-inflammatory and antinociceptive activities A of eugenol essential oil in experimental animal models. Revista Brasileira de Farmacognosia, 19, $212-217$.

Dardes, R. D. C. D. M., Moraes, A. S., \& Santos, M. B. (2011). Dismenorreia. RBM rev. bras. med.

Dias, S. F. L., Pereira, L. C. A., Oliveira, A. P. D., Santos, R. F. D., \& Nunes, L. C. C. (2019). Scientific and technological prospection on transdermal formulations and complementary therapies for the treatment of primary dysmenorrhea. Expert opinion on therapeutic patents, 29 (2), $115-126$.

Diegoli, C. A., \& Diegoli, M. S. C. (2007). Saúde da Mulher Dismenorréia. Revista Brasileira de Medicina-RBM, 64 (3).

Dos Santos, G. K. A., Silva, N. C. D. O. V., \& Alfieri, F. M. (2020). Efeitos da compressa fria versus quente sobre a dor em universitárias com dismenorreia primária. $\operatorname{BrJP}, 3,25-28$.

Fae, A., \& Pivetta, H. M. F. (2010). Efeitos da massagem do tecido conjuntivo e cinesioterapia no tratamento da dismenorréia primária. Fisioterapia Brasil, 11 $(2)$

Fonseca, A. M., \& Bagnoli, V. R. (2004). Como diagnosticar e tratar: dismenorréia. Revista Brasileira de Medicina (Rio de Janeiro), 62, 113-6.

Frare, J. C., Tomadon, A., \& Silva, J. R. (2014). Prevalência da dismenorreia e seu efeito na qualidade de vida entre mulheres jovens. Revista Brasileira de Ciências da Saúde, 12 (39), 15-20.

Gaubeca-Gilarranz, A., Fernández-De-Las-Peñas, C., Medina-Torres, J. R., Seoane-Ruiz, J. M., Company-Palonés, A., Cleland, J. A., \& Arias-Buría, J. L. (2018). Effectiveness of dry needling of rectus abdominis trigger points for the treatment of primary dysmenorrhoea: a randomised parallel-group trial. Acupuncture in Medicine, 36 (5), 302-310.

Ghods, A. A., Abforosh, N. H., Ghorbani, R., \& Asgari, M. R. (2015). The effect of topical application of lavender essential oil on the intensity of pain caused by the insertion of dialysis needles in hemodialysis patients: a randomized clinical trial. Complementary therapies in medicine, 23 (3), $325-330$.

Hanfy, H. M., Kamel, H. E. H., Kamal, W. M., \& Mahmoud, S. M. (2020). The effect of progressive relaxation training versus aromatherapy on primary dysmenorrhea. Egypt, Med. J. Cairo Univ, 88 (2), 577-582.

Hoare, J., \& Wilson, S. (2010). Guia completo de aromaterapia: um curso estruturado para alcançar a excelência profissional. Pensamento.

Hur, M. H., Lee, M. S., Seong, K. Y., \& Lee, M. K. (2012). Aromatherapy massage on the abdomen for alleviating menstrual pain in high school girls: a preliminary controlled clinical study. Evidence-Based Complementary and Alternative Medicine, 2012.

Jun, W. J., Han, B. K., Yu, K. W., Kim, M. S., Chang, I. S., Kim, H. Y., \& Cho, H. Y. (2001). Antioxidant effects of Origanum majorana L. on superoxide anion radicals. Food Chemistry, 75 (4), 439-444.

Kartikasari, R. (2020). Effect Of Peppermint Aromatherapy On Menstruation Scale Of Pain In Fakultas Kedokteran Hang Tuah Surabaya Students. Jurnal Agri-Tek: Jurnal Penelitian Ilmu-Ilmu Eksakta, 21 (1), 10-13.

Kim, Y. J., Lee, M. S., Yang, Y. S., \& Hur, M. H. (2011). Self-aromatherapy massage of the abdomen for the reduction of menstrual pain and anxiety during menstruation in nurses: a placebo-controlled clinical trial. European journal of integrative medicine, 3 (3), e165-e168.

Klotz, U. (2012). Paracetamol (acetaminophen)-a popular and widely used nonopioid analgesic. Arzneimittelforschung, 62 (08), $355-359$.

Lee, M. S., Choi, J., Posadzki, P., \& Ernst, E. (2012). Aromatherapy for health care: an overview of systematic reviews. Maturitas, 71 (3), 257-260.

Lefebvre, G., Pinsonneault, O., Antao, V., Black, A., Burnett, M., Feldman, K., ... \& Robert, M. (2005). Primary dysmenorrhea consensus guideline. J Obstet Gynaecol Can, 27 (12), 1117-46.

Leite, M. C. A., Leite, C. A., Machado, R. F. F., Portella, A. S. J. A., \& Simões, B. N. (2010). Dismenorréia: uma visão atual. Anais do, 11.

Leite, M. C. A., \& da Nóbrega, M. M. (2008). A dismenorréia primária como problema de saúde pública. Revista de Ciências da Saúde Nova Esperança, 6 (1), 8-19.

Loulanski, V., \& Loulanski, T. (2014). The heritization of Bulgarian rose. Acta Geographica Slovenica, 54 (2), $401-410$. 
Marzouk, T. M., El-Nemer, A. M., \& Baraka, H. N. (2013). The effect of aromatherapy abdominal massage on alleviating menstrual pain in nursing students: a prospective randomized cross-over study. Evidence-Based Complementary and Alternative Medicine, 2013.

Metzger, C., Schwetta, M., Walter, C., Müller, A., \& Martins, M. M. S. (2002). Cuidados de Enfermagem e dor.

Miot, H. A. (2011). Tamanho da amostra em estudos clínicos e experimentais. Jornal Vascular Brasileiro, 10, $275-278$.

Montibeler, J., Domingos, T. D. S., Braga, E. M., Gnatta, J. R., Kurebayashi, L. F. S., \& Kurebayashi, A. K. (2018). Efetividade da massagem com aromaterapia no estresse da equipe de enfermagem do centro cirúrgico: estudo-piloto. Revista da Escola de Enfermagem da USP, 52.

Nicácio, G. L., Moura, S. C., Costa, J. V. D. J., Sena, C. R., Cruz, T. B. F., Lopes, G. N. M., \& Cecílio, A. B. (2018). Breve revisão sobre as propriedades fitoterápicas do Zingiber officinale Roscoe - o gengibre. Sinapse múltipla, 7 (2), 74-80.

Oliveira Nunes, J. M., do Amaral Rodrigues, J., de Freitas Moura, M. S., Batista, S. R. C., Coutinho, S. K. S. F., Hazime, F. A., \& dos Reis Barbosa, A. L. (2013). Prevalência de dismenorreia em universitárias e sua relação com absenteísmo escolar, exercício físico e uso de medicamentos. Revista Brasileira em Promoção da Saúde, 26 (3), 381-386.

Ou, M. C., Hsu, T. F., Lai, A. C., Lin, Y. T., \& Lin, C. C. (2012). Pain relief assessment by aromatic essential oil massage on outpatients with primary dysmenorrhea: A randomized, double-blind clinical trial. Journal of Obstetrics and Gynaecology Research, 38 (5), $817-822$

Paredes, J. S., Jaramillo, A. M., \& Paz, C. S. P. S. (2013). Factores que condicionan la dismenorrea en adolescentes, según estrato socioeconómico. Revista Peruana de Ginecología y obstetricia, 59(2), 95-100.

Paviani, B. A., Trigueiro, T. H., \& Gessner, R. (2019). O uso de óleos essenciais no trabalho de parto e parto: revisão de escopo. Revista Mineira de Enfermagem, 23, 1-8.

Pereira, A., Bertoni, B., Silva, C., Ferro, D., Carmona, F., Dandaro, I., ... \& Doneida, V. (2017). Formulário de preparação extemporânea: farmácia da natureza-chás medicinais.

Price, S., \& Price, L. (2011). Aromatherapy for health professionals E-book. Elsevier Health Sciences.

Pujiati, W., Siagian, Y., \& Hardivianty, C. (2018). Application of Essential Oils: Lavender, Clary Sage, Ginger and Geranium as Aromatherapy through Effleurage Massage for Menstrual Pain. International Journal of Science and Research (IJSR).

Rizk, S. A. (2013). Effect of aromatherapy abdominal massage using peppermint versus ginger oils on primary dysmenorrhea among adolescent girls. Journal of American Science, 9 (11), 497-505.

Rodrigues, A. C., Gala, S., Neves, A., Pinto, C., Meirelles, C., Frutuoso, C., \& Vítor, M. E. (2011). Dismenorreia em adolescentes e jovens adultas: prevalência, factores associados e limitações na vida diária. Acta Med Port, 24 (S2), 383-92.

Rodrigues, M. L., \& Lira, R. K. (2013). Perfil fitoquímico e biológico do extrato hidroalcoólico dos rizomas do gengibre (Zingiber officinale Roscoe). SaBiosRevista de Saúde e Biologia, 8 (1).

Sacco, P. R., Ferreira, G. C. G. B., \& Silva, A. C. C. D. (2015). Aromaterapia no auxílio do combate ao estresse: bem-estar e qualidade de vida. Revista científica da FHO| UNIARARAS, 3 (1).

Sajjadi, M., Bahri, N., \& Abavisani, M. (2018). Aromatherapy massage with geranium essence for pain reduction of primary dysmenorrhea: a double blind clinical trial. The Iranian Journal of Obstetrics, Gynecology and Infertility, 20 (12), 50-57.

Sezeremeta, D. C., dos Santos Carvalho, M. S., Vrecchi, M. R., Marafon, R. G. C., Crespilho, L. C., Pagotto, J. P., \& Mortean, E. D. C. M. (2013). Dismenorreia: Ocorrência na vida de acadêmicas da área de saúde. Journal of Health Sciences, 15 (2).

Shahr, H., Saadat, M., Kheirkhah, M., \& Saadat, E. (2015). The effect of self-aromatherapy massage of the abdomen on the primary dysmenorrhoea. Journal of Obstetrics and Gynaecology, 35 (4), 382-385.

Shokri, H., Khosravi, A. R., Mansouri, M., \& Ziglari, T. (2011). Effects of Zataria multiflora and Geranium pelargonium essential oils on growth-inhibiting of some toxigenic fungi. Iranian Journal of Veterinary Research, 12(3), 247-251.

Siddaway ,AP, Wood AM, Hedges LV.(2019)How to do a Systematic review: a best practice guide for conduncting and reporting narrative reviews, metaanalyses, and meta-syntheses.Annu Ver Psychol;70:747-70

Silva, A. B., Pereira, A. O., Silva, S. P., Lima, C. R. J., \& Lima, A. B. (2012). Correlação entre as alterações posturais e a dismenorreia primária em mulheres jovens na faixa etária de 18 a 25 anos. Revista Científica do Unisalesiano-Lins-SP, 3 (6), 253-265.

Sinha, A. G. (2010). Principle and practice of therapeutic massage. Editor: Textbook of massage. India: Jaypee Brothers Medical Publishers, p.8-23. Strehl, L, Calabró L, Souza, DO, Amaral, L(2016)Brazilian Science between National and Foreig Journais: Methodology for Analyzing the Producion and Impact in Emerging Scientific Communities. PLoS ONE. 2016; 11 (5): e0155148. Doi: 10.1371/journal.pone,0155148

Sujana, P., Sridhar, T. M., Josthna, P., \& Naidu, C. V. (2013). Antibacterial activity and phytochemical analysis of Mentha piperita L.(Peppermint) - An importante multipurpose medicinal plant. Am J Plant Sci; 4 (1):77-83.

Tobo, A., Khouri, M., Cordeiro, Q., Lima, M. D. C., Brito Junior, C. A., \& Battistella, L. R. (2010). Estudo do tratamento da lombalgia crônica por meio da Escola de Postura. Acta fisiatrica, 17 (3), 112-6.

Yassin, S. A. (2012). Herbal remedy used by rural adolescent girls with menstrual disorders. J Am Sci, 8 (1), 467 - 